\title{
Towards an Air Mobile Stroke Unit for Rapid Medical Response in Rural Australia
}

\author{
Cees BIL ${ }^{\mathrm{a}, 1}$, Silke WALTER ${ }^{\mathrm{b}}$, Jonas SAUER ${ }^{\mathrm{a}}$ and Sebastian FELDMANN ${ }^{\mathrm{a}}$ \\ ${ }^{a}$ RMIT University, School of Engineering, Melbourne, Australia \\ ${ }^{\mathrm{b}}$ Saarland University Medical Center, Homburg, Germany
}

\begin{abstract}
Stroke is highly treatable but time critical. The greatest opportunity to improve outcomes is in the first 'Golden Hour' after onset. Pre-hospital care for stroke in Australia is patchy and poorly coordinated, resulting in gross disparities in clinical outcomes between rural and urban Australians. Clinical outcomes are at least twice as poor for rural Australians compared to their urban counterparts. This paper discusses a transformative research program that investigates the technical feasibility of transporting a CT-scan device to the patient to determine the cause of stroke and initiate treatment immediately. This concept follows in the tradition of the Royal Flying Doctors Service who have been providing medical service to rural Australians since 1928.
\end{abstract}

Keywords. Stroke, Medical Service, Golden Hour, Air Mobile Stroke Unit, Rural Area

\section{Introduction}

Worldwide, there are over 6 million deaths annually due to stroke. In Ausralia, the number of strokes will increase from 60,000 to 132,500 per year by 2050 . This represents an enormous economic burden, particularly with an ageing population. Stroke is highly treatable but time critical. The greatest opportunity to improve outcomes is in the first 'Golden Hour' after onset. Pre-hospital care for stroke in Australia is patchy and poorly coordinated, resulting in gross disparities in clinical outcomes between rural and urban Australians. The incidence of stroke is approximately $20 \%$ higher in rural areas, particularly for Indigenous Australians where life expectancy which is already 10 years shorter. Clinical outcomes are at least twice as poor for rural Australians compared to their urban counterparts. To optimise the pre-hospital treatment of stroke, a transformative research program is proposed to develop, test and implement novel disruptive technologies. The aim is to reduce morbidity and mortality, and narrow the urban, rural and Indigenous healthcare gaps. Part of this research program is the development of a road and air Mobile Stroke Unit (MSU) that can bring diagnostic and treatment equipment to the patient.

Stroke is a primary cause of death, disability, and dementia $[1,2]$. In the next decades the prevalence of stroke is expected to increase because of the increase of the aged population in most countries [3]. Apart from considerable suffering for the patients and their families, stroke also results in enormous costs to society, costs associated with loss of work hours and with long-term care expenses [4,5].

\footnotetext{
${ }^{1}$ Corresponding Author, Email: c.bil@rmit.edu.au
} 


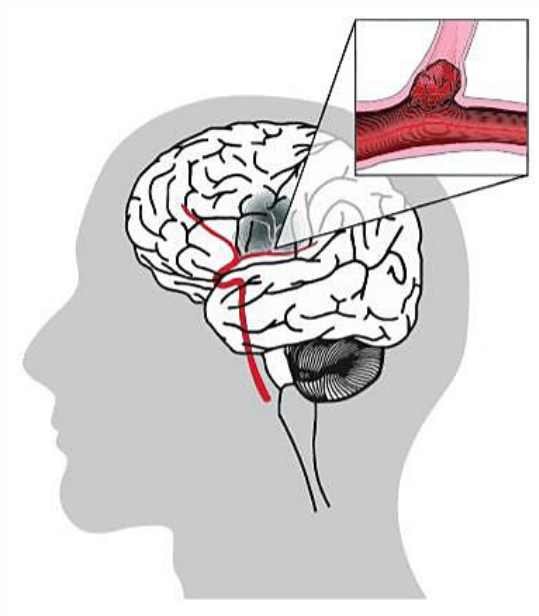

Ischaemic stroke

(embolic and

thrombotic)



Haemorrhagic stroke

(subarachnoid and intracerebral)

Figure 1. Ischaemic stroke and haemorrhagic stroke (National Stroke Foundation 2014).

Basically, two major forms of stroke can be distinguished (Figure 1). An ischaemic stroke is characterized by blocking (clots) of an artery transporting blood to parts of the brain. The second kind of stroke is the haemorrhagic stroke, caused by bursting of a blood vessel within the brain and floods the surrounding area. Due to the pressure in the artery the rupturing blood can damage the sensitive brain tissue. Usually it is impossible to identify the stroke type on the basis of symptoms. Therefore, imaging tests of the brain are recommended to determine the stroke type and the extent of damage. Those scans can be performed by either a Computerised Tomography (CT) scan using X-rays for producing 2- or 3-dimensional images or by a Magnetic Resonance Imaging (MRI) scan which is based on generating a magnetic field and low-energy radio waves also used for producing 2- or 3-dimensional images. In general, a CT is less sensitive than an MRI but sufficient for the identification of an ischaemic stroke [6].

After the diagnosis of a stroke the patient should be treated as soon as possible to avoid further damages of the brain. Acute ischemic stroke is treatable by thrombolysis with recombinant tissue Plasminogen Activator (rtPA) or by removal of the clot in the brain-supplying vessel by Intra-Arterial Treatment (IAT), in case of Large Vessel Occlusion (LVO). Both types of recanalizing treatments must be delivered as quickly as possible after the onset of stroke symptoms because the chances of rescuing the ischemic brain from irreversible damage decline rapidly and even minutes of delay count $[7,8]$. Haemorrhagic stroke is treated by emergency surgery in order to remove the accumulated blood coming out of the ruptured vessel. Simultaneously the blood pressure needs to be reduced by using medication [9].

\section{Mobile Stroke Unit concept}

Timely delivery of recanalizing stroke treatment is a serious medical problem, especially in countries with large remote areas and low population densities, in which coverage 
with specialized stroke centres is poor. Within a single country, the rural or urban setting and the associated distances to nearest stroke centre are key factors in the delivery of time-sensitive stroke treatment [10,11]. Reported times from symptom onset to admission to rural hospitals range from 5 hours to 30 hours [12,13]. In Australia, for example, the likelihood that patients in rural areas could access a stroke unit in time was only $3 \%$, whereas the one of patients in metropolitan areas was $77 \%$ [14]. These delays before hospital admission are a major reason for low thrombolysis rates of $1 \%$ to $6 \%$ for stroke patients in rural areas in different countries [15-18]. Such a disparity in regard to acute stroke treatment is a major medical problem not only in low-income countries but also in middle- and high-income countries, such as Brazil, Russia, the United States, Canada, and Australia [12,15-18].

This treatment gap is even more pronounced with regard to the delivery of the novel highly effective endovascular treatment options for acute stroke [19]. Importantly, delivery of IAT requires substantial human and technical resources with highly specialized endovascular teams and additional peri-interventional services (neurosurgery, anaesthesiology, and neurointensive care). Such complex infrastructure is available only on a 24/7 level only in a few Comprehensive Stroke Centres (CSC), which are usually located in metropolitan, not rural, areas [20]. Patients living in rural remote regions have very limited access to advanced stroke treatments, if at all [21]. In the recent years, awareness increases about the detrimental treatment gap for stroke patients living in remote regions and about the urgent need for novel systems of care to overcome this disparity [22].

A recently developed concept is the provision of diagnosis and treatment of stroke already at the emergency site. This is based on an ambulance (Mobile Stroke Unit, MSU) equipped, apart from the conventional ambulance equipment, with all of the tools necessary for prehospital diagnosis and treatment and a rational triage decision with regard to most appropriate target hospital: A computed tomography (CT) scanner for multimodal imaging studies, a point-of-care (POC) laboratory for all blood tests required for treatment in accordance with approval criteria [23] and stroke management guidelines $[24,25]$ and a telemedicine connection to hospital stroke experts.

Several trials have shown that this approach significantly reduces delays before intravenous (IV) thrombolysis [26-29]. The rate of patients treated within 60 min after the onset of stroke symptoms ("the golden hour of stroke") increased dramatically, from $4 \%$ to $57 \%$ [26]. This reduction in delay before treatment is due not only to a reduction in transport times but also to the marked improvement in efficiency at the interfaces between the many groups of health care professionals involved in stroke management [30].

In addition, diagnostic clarification of the vascular pathology directly at the emergency site by prehospital CT angiography in the ambulance enables, for the first time, an etiology-based triage decision with regard to the most appropriate target hospital (CSC, regional hospital or primary stroke centre [PSC]) to which the patient should be transported. On the one hand, patients should not be taken to a hospital without thrombectomy capabilities. On the other hand, not all patients can be transferred to a CSC, which would impair the efficient use of their limited resources, considering that most patients are not eligible for IAT [22]. The MSU concept may solve this problem, because, apart from prehosptial thrombolysis, it enables a rational triage decision in regard to the appropriate target hospital already at the emergency site. 


\subsection{Road Mobile Stroke Unit}

First publications about a MSU date back to the year 2010, when a regular road ambulance was converted into the first MSU. Most important additional hardware needed was a CT-scanner, a point of care laboratory and a telemedicine connection (Figure 2) [30]. The telemedicine connection provides access to the expertise of a vascular neurologist and it is sufficient when only paramedics are on-board the MSU [31].
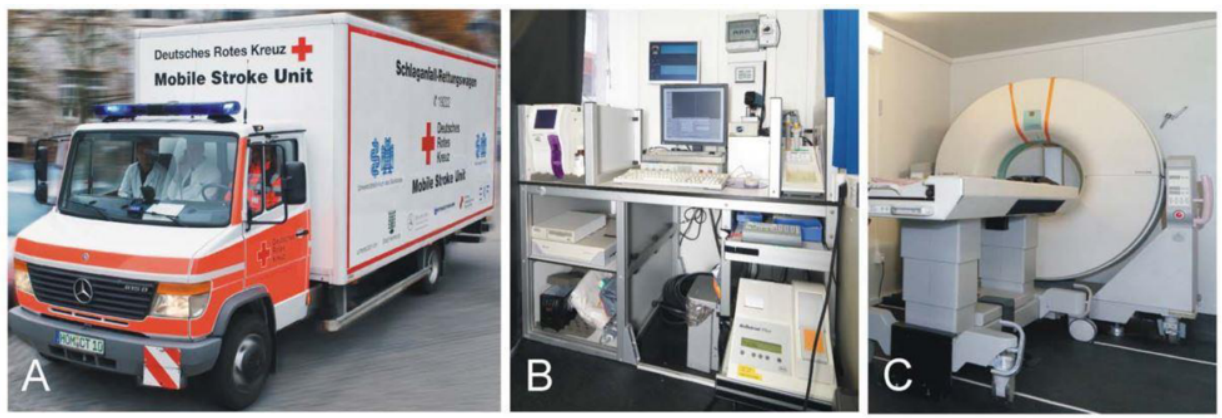

Figure 2. An ambulance (A) equipped with point-of-care laboratory system and telemedicine devices (B) and $\mathrm{CT}$ (C) required for pre-hospital stroke treatment [30].

For the two patients, which were treated in the first trial, the time between symptoms onset and treatment was reduced significantly [30]. Because the treatment can already start inside the MSU, another time benefit occurs as all diagnostic work and treatment can be done directly at the emergency site in a parallel workflow. In addition, the patient can afterwards be taken straight into a specialized stroke centre, so the patient only needs to be transported once as shown in Figure 3 [31]. In a trial with one hundred stroke patients the time between alarm and therapy decision was roughly halved [26]. It was shown that the MSU is reliable for daily use but that the CT-scanner needs regular preparation and maintenance. The use of road MSUs is increasing worldwide and has reached more than twenty sites so far [30]. Melbourne received its first road MSU in 2017 [32].

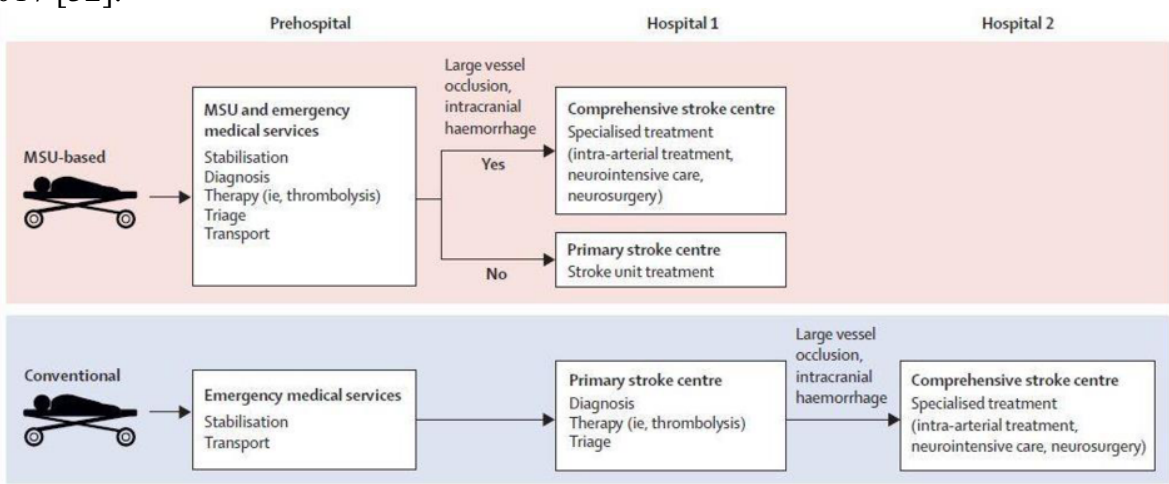

Figure 3. MSU-based stroke management compared with conventional stroke management [30]. 


\section{MSU Saarland}

The basic principle of a MSU is bringing all diagnostic tools, treatment devices and medicines directly to the emergency site to reduce the "door to needle time". The world first road MSU started its operation in 2008 in the Saarland (Germany) after 5 years of development. Two years later, the operators of the MSU confirmed the feasibility of the project. Fassbender et al. compared the therapy decision times of patients treated in the MSU (intervention group) and a control group consisting of patients being treated conventionally in hospital [31]. The results show that $57 \%$ of the patients in the intervention group but only $5 \%$ of the patients in the control group were treated within one hour. The Saarland MSU is an road ambulance equipped with the standard medical equipment, a battery-driven CT-scanner, a telemedicine system and a point-of-care laboratory. It is staffed with a paramedic, a physician and an optional neuroradiologist as the telemedicine system might replace the neuroradiologist [30].

\section{MSU Melbourne}

The fact that a new stroke occurs in Australia every 10 minutes might have led to the launch of the Victorian Stroke Telemedicine Program. The objective of this program is to provide people living in rural areas access to stroke specialists. The first Australian Mobile Stroke Unit in Melbourne in 2017 is part of this program. Like the Saarland project, the Melbourne MSU is equipped with a CT scanner allowing brain imaging at the emergency site. The team working on the ambulance consists of a stroke nurse, radiographer, neurologist and a paramedic [32].

\section{MSU USA}

The first MSU in the United States was introduced in 2014. Major obstacles during the development time were obtaining funding, licensure, radiation safety protocols and a smooth communication system [33]. Significant modifications had to made to the vehicle to make it compatible with the CT scanner. After the reconfiguration was finished and the MSU had operated for some weeks the number of patients treated within 90 minutes could be increased from $37 \%$ to $58 \%$ [33].

\subsection{Air Mobile Stroke Unit}

Although stroke treatment is very time-sensitive, only 3\% of Australian stroke patients in rural areas get treated in a timely manner. In the metropolitan region $77 \%$ patients get treated within this time because the intra-arterial therapy services are not as limited as in the rural areas. Because of a treatment gap for stroke patients in rural and remote areas suggest the use of specially designed aircraft, the so-called Air Mobile Stroke Units (Air MSU) in these areas [34]. The Air MSU offers the ability to diagnose and treat acute stroke patients directly at the emergency site with the help of an onboard computed tomography (CT) scanner, a point-of-care blood laboratory and telemedicine connection to a stroke specialist. The use of such an MSU reduces the door-to-needle time significantly and increases treatments within 60 minutes after stroke occurrence ("golden hour") from $4 \%$ to $57 \%$. Moreover, inter-hospital transfers can be avoided as the next, most suited medical centre can be chosen depending on the results of the CT scan which would be performed in advance at the emergency site. For the evaluation of the CT scan the telemedicine system, which is a video conference with an expert including the exchange of videos and images, can be used during or after scanning [34]. 


\section{Air MSU missions}

Two main scenarios can be anticipated in use of an Air MSU in order to improve access to state-of-the art stroke treatment in remote areas (Fig. 4):

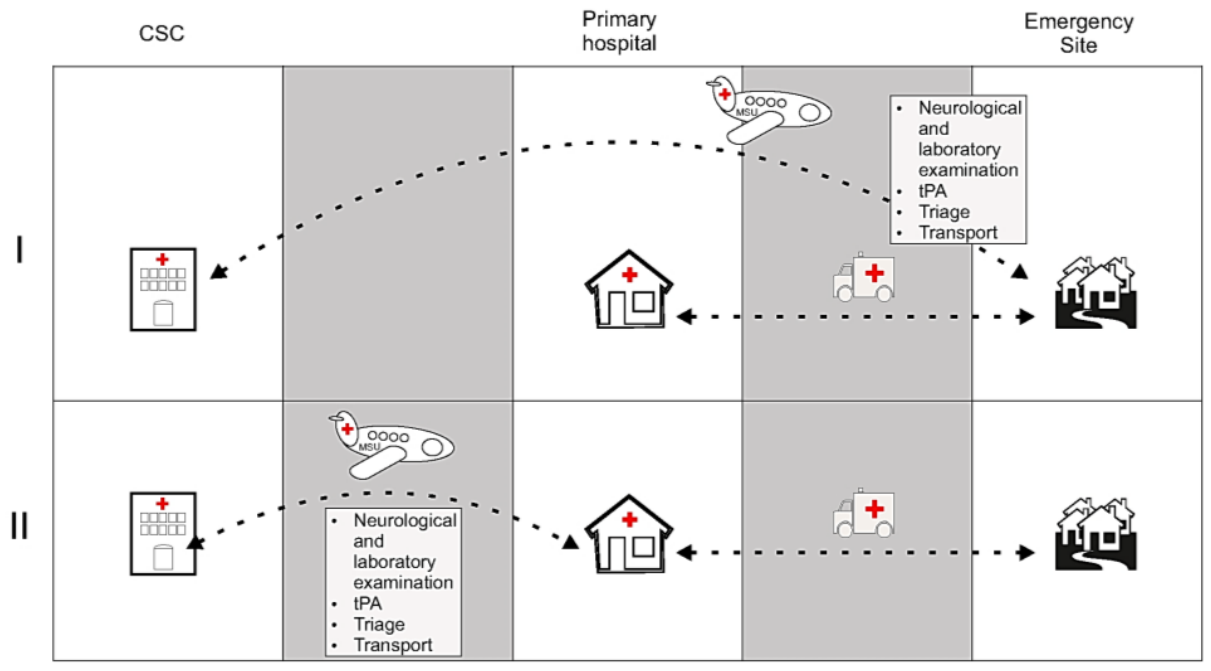

Figure 4. Two scenarios of Air-Mobile Stroke Unit (MSU) missions.

In scenario 1, the Air MSU, having been activated by the Emergency Medical Services (EMS) dispatch centre, reaches the patient at the emergency site, using the closest possible landing option. There, all stroke examinations are performed already in the pre-hospital phase of stroke management, and, if indicated, treatment is initiated in the field. Moreover, based on the vascular cause, a correct triage decision about the appropriate target hospital can be made. In case of a haemorrhage, the Air MSU may, after pre-notification, transfer the patient directly to a CSC for required endovascular, neurosurgical or neurointensive treatment. All other cases may be triaged to the nearest regional hospital. The Air MSU can function as a primary EMS vehicle or meets the first responding EMS.

In scenario 2, the patient has been admitted to a primary hospital without thrombectomy capability. The alerted Air MSU team would come to take over the specialized stroke management. This includes, apart from indicated IV thrombolysis, a vascular diagnostic work up by CT angiography and perfusion, as a pre-condition for a rational triage decision about whether or not the patient should be transferred to a CSC. Alternatively, in a rural hospital the patient could, while the Air MSU is underway, obtain first neurological assessment and, after non-contrast imaging, thrombolytic treatment. After arrival, the Air MSU would complement stroke management with vascular imaging as a pre-condition for a rational triage decision, and, if indicated, realizes the transport of the patient to the CSC for specialized stroke treatment. 


\subsection{Air MSU Design Challenges}

Helicopter Emergency Medical Services (HEMS) play an important role in today's trauma patient transportation. The main benefits come from rapid transportation of small group of experts capable of advanced lifesaving procedures, and the direct transport to a specialized trauma center avoiding a second transportation [35]. A study shows that $96.4 \%$ of stroke patients transported with HEMS arrive within two hours of dispatch request, $99.9 \%$ arrive within 4.5 hours [36]. That classifies HEMS as an appropriate mode of transportation for stroke patients [37]. Patients transported by HEMS show a significant survival benefit compared with road transport $[35,38]$. However, only $28 \%$ of HEMS in the USA are operating between emergency sites and hospital, while $72 \%$ represent inter-hospital transfer [36]. Studies show that for inter-hospital transportation HEMS is faster than road, while another study suggests that HEMS is only faster the travelling distance is more than $50 \mathrm{~km}[39,40]$. Table 1 shows some of the advantages and disadvantages for helicopter versus road medical transportation [40].

Table 1. Advantages and disadvantages of road ambulenance versus helicopter ambulance [40].

\begin{tabular}{|c|c|c|}
\hline & Ground Ambulance & Helicopter Ambulance \\
\hline Human expertise & $\begin{array}{l}\text { EMTs, voluntary firefighters } \\
\text { (rural areas), physicians (outside } \\
\text { USA) }\end{array}$ & $\begin{array}{l}\text { EMT, nurses } \\
\text { physicians (in-flight), } \\
\text { extenders (local ED), stroke } \\
\text { specialists (phone/telemedicine) }\end{array}$ \\
\hline Speed & + & +++ \\
\hline $\begin{array}{l}\text { Vulnerability } \\
\text { weather/altitude/humidity }\end{array}$ & + & +++ \\
\hline Weight restrictions & - & +++ \\
\hline Space limitations & + & +++ \\
\hline CT-carrying capability & yes & no \\
\hline $\begin{array}{l}\text { Potential impact on physical } \\
\text { factors on stroke patients }\end{array}$ & + & +++ \\
\hline Cost & + & +++ \\
\hline Availability & +++ & + \\
\hline
\end{tabular}

\subsection{Helicopter selection}

Table 2 shows some additional helicopter information, such as range and cruise speed. While the helicopters with a smaller cabin are often used for EMS, larger helicopters are mainly for Search and Rescue (SAR).

Table 2. Technical comparison of existing helicopter types [44].

\begin{tabular}{ccccccc}
\hline $\begin{array}{c}\text { Helicopter } \\
\text { Type }\end{array}$ & EMS & $\begin{array}{c}\text { Cruise Speed } \\
(\mathrm{km} / \mathrm{hr})\end{array}$ & $\begin{array}{c}\text { Max } \\
\text { Range } \\
(\mathrm{km})\end{array}$ & $\begin{array}{c}\text { MTOW } \\
(\mathrm{kg})\end{array}$ & $\begin{array}{c}\text { Payload } \\
\text { Weight } \\
(\mathrm{kg})\end{array}$ & $\begin{array}{c}\text { Cabin }(\mathrm{m}) \\
\mathrm{H} \times \mathrm{W} \times \mathrm{L}\end{array}$ \\
\hline AW-139 & yes & 306 & 1061 & 6,400 & 2,778 & $1.42 \times 2.08 \times 2.79$ \\
EC-155 & yes & 270 & 791 & 4,920 & 2,302 & $1.34 \times 2.05 \times 2.55$ \\
S-76D & yes & 287 & 874 & 5,386 & 2,221 & $1.35 \times 1.93 \times 2.41$ \\
Bell 412 EPI & yes & 235 & 687 & 5,398 & 2,191 & $1.35 \times 2.43 \times 2.34$ \\
AW-101 & no (SAR) & 277 & 1500 & 15,600 & 5,443 & $1.83 \times 2.50 \times 6.50$ \\
AW-189 & no (SAR) & 287 & 1111 & 8,300 & 2,675 & $1.40 \times 2.40 \times 3.40$ \\
EC-225 & no (SAR) & 275 & 1185 & 11,000 & 5,624 & $1.45 \times 1.80 \times 5.69$ \\
S-92 & no (SAR) & 280 & 1185 & 12,020 & 3,897 & $1.83 \times 2.01 \times 6.10$ \\
NH-90 & no (military) & 300 & 982 & 10,600 & 4,200 & $1.58 \times 2.00 \times 4.80$ \\
\hline
\end{tabular}


A critical selection criteria is the accommodation of the CT-scanner in the cabin. Typical hospital mobile CT-scanners are about $1.4 \mathrm{~m}$ high which eliminates some of the smaller helicopters. For a typical MSU mission, the payload weight is between 1,500 $\mathrm{kg}$ to $2,000 \mathrm{~kg}$, depending on the equipment Table 3. Typical payload for Air MSU operations. carried (Table 3 ).

The typical weight of a scanner is between $500 \mathrm{~kg}-700 \mathrm{~kg}$ which takes up a significant amount of payload weight. A typical layout of an aircraft cabin for Air MSU is shown in Figure 5 [44].
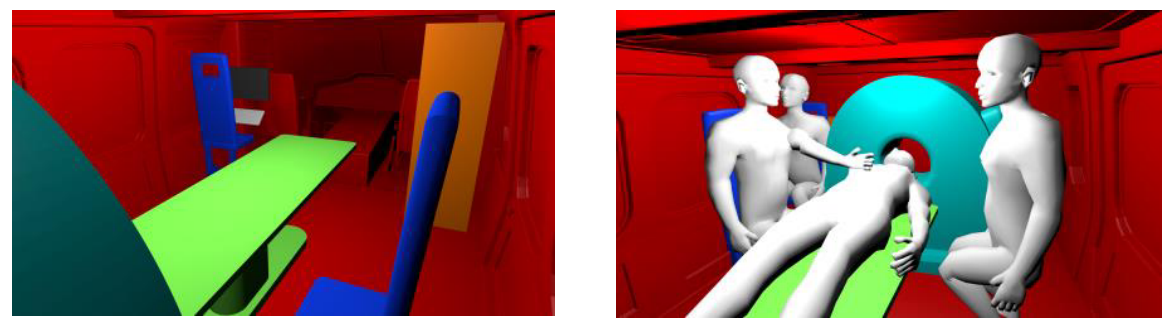

Figure 5. Typical Air MSU helicopter cabin layout.

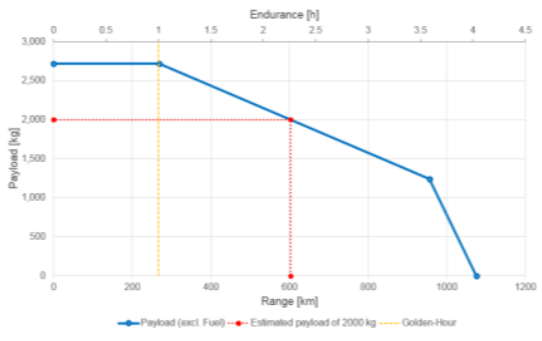

Figure 6. Payload - Range diagram.

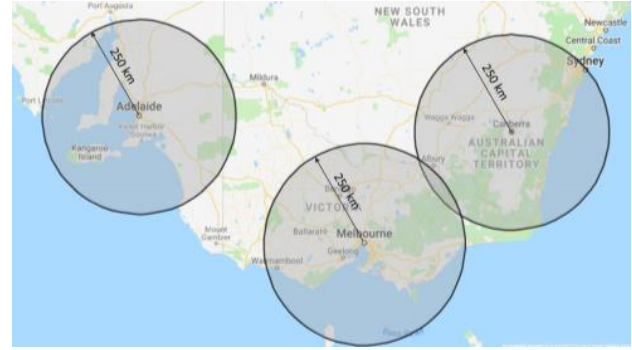

Figure 7. Area coverage at $250 \mathrm{~km}$ range (Golden Hour).

Figure 6 shows the payload-range diagram that gives the range and endurance capability as a function of payload weight. Effective range will be reduced to maintain a maximum $60 \mathrm{~min}$ response time. Figure 7 shows that coverage of the State of Victoria for a $250 \mathrm{~km}$ range (one-way), based on $1 \mathrm{hr}$ flight time (Golden Hour). This shows that a number of Air MSUs are needed to be placed strategically to cover the state.

\section{Conclusion}

Novel health care solutions are urgently needed that reduce the treatment gap for stroke patients living in rural, remote regions. This is especially true with regard to the poor access to the novel endovascular stroke treatment options of rural stroke patients. The Air-MSU may reduce this treatment disparity although this approach requires substantial research regarding the optimal technical solutions, the best setting, the ideal radius of 
dispatch, the best way of integration into the routine ground- or air-borne EMS, the effects on long-term outcomes, and the cost-efficiency in comparison with standard care in these settings. Research is required to analyse whether costs associated with worse outcomes associated with poor access to advance stroke treatment can outweigh costs associated with additional resources of an Air MSU. This also depends on potential synergistic effects with already existing flying doctor services exits and extension of the indications of this concept in regard to further time-sensitive emergencies such as brain trauma or myocardial infarction.

\section{References}

[1] B. Ovbiagele, L.B. Goldstein, R.T. Higashida et al. American Heart Association Advocacy Coordinating Committee and Stroke Council. Forecasting the future of stroke in the United States: a policy statement from the American Heart Association and American Stroke Association. Stroke, 2013, Vol. 44, pp. 23612375 .

[2] Writing Group Members, Mozaffarian D, Benjamin EJ, Go AS et al; American Heart Association Statistics Committee; Stroke Statistics Subcommittee. Heart disease and stroke statistics-2016 update: a report from the American Heart Association. Circulation 2016; 133:e38-360.

[3] V.L. Feigin, B. Norrving, G.A. Mensah, Global burden of stroke. Circ Res, 2017; 120:439-48.

[4] J. Olesen, A. Gustavsson, M. Svensson, H.U. Wittchen and B. Jönsson; CDBE2010 study group; European Brain Council. The economic cost of brain disorders in Europe. Eur J Neurol, 2012; 19:15562.

[5] V.L. Roger, A.S. Go, D.M. Lloyd-Jones et al; American Heart Association Statistics Committee and Stroke Statistics Subcommittee. Heart disease and stroke statistics--2012 update: a report from the American Heart Association. Circulation 2012; 125:e2-220.

[6] Garcia-Esperon, NSC 2017, Stroke, Brain Foundation, accessed Oct 14, 2018, $<$ https://brainfoundation.org.au/disorders/stroke $>$.

[7] J. Emberson, K.R. Lees, P. Lyden et al; Stroke Thrombolysis Trialists' Collaborative Group. Effect of treatment delay, age, and stroke severity on the effects of intravenous thrombolysis with alteplase for acute ischaemic stroke: a meta-analysis of individual patient data from randomised trials. Lancet, 2014; 384:1929-35.

[8] J.L. Saver, Time is brain--quantified. Stroke, 2006; 37:263-6.

[9] A. Mandal, 2018, Stroke Treatment, News-Medical.net, 15.10.2018, <https://www.newsmedical.net/health/Stroke-Treatment.aspx>.

[10] A.M. Cox, C. McKevitt, A.G. Rudd, C.D. Wolfe, Socioeconomic status and stroke. Lancet Neurol, 2006; 5:181-8.

[11] Y. Teuschl, M. Brainin, Stroke education: discrepancies among factors influencing prehospital delay and stroke knowledge. Int J Stroke, 2010; 5:187-208.

[12] J. Joubert, L.F. Prentice, T. Moulin et al. Stroke in rural areas and small communities. Stroke, 2008; 39:1920-8.

[13] J. Oliveira-Filho, S.C. Martins, O.M. Pontes-Neto et al; Executive Committee from Brazilian Stroke Society and the Scientific Department in Cerebrovascular Diseases. Guidelines for acute ischemic stroke treatment: part I. Arq Neuropsiquiatr, 2012; 70:621-9.

[14] A. Eissa, I. Krass, B.V. Bajorek, Optimizing the management of acute ischaemic stroke: a review of the utilization of intravenous recombinant tissue plasminogen activator (tPA). J Clin Pharm Ther, 2012; 37:620-9.

[15] D.A. Cadilhac, T. Purvis, M.F. Kilkenny et al; New South Wales Strokes Services Coordinating Committee; Agency for Clinical Innovation. Evaluation of rural stroke services: does implementation of coordinators and pathways improve care in rural hospitals? Stroke, 2013; 44:2848-53.

[16] L.V. Stakhovskaia, K.V. Shekhovtsova, T.I. Rozhkova et al., Medical service to patients with stroke at the prehospital stage in different cities of the Russian Federation, Zh Nevrol Psikhiatr, 2010; 110:36-41.

[17] A. Ganesh, M. Camden, P. Lindsay et al; Canadian Stroke Audit Group. The quality of treatment of hyperacute ischemic stroke in Canada: a retrospective chart audit. CMAJ Open, 2014; 2:E233-9.

[18] J. Newbury, T. Kleinig, J. Leyden et al. Stroke Epidemiology in an Australian Rural Cohort (SEARCH). Int J Stroke, 2017; 12:161-8.

[19] S. Prabhakaran, I. Ruff, R.A. Bernstein, Acute stroke intervention: a systematic review. JAMA, 2015; 313:1451-62. 
[20] B.K. Menon, B.C. Campbell, C. Levi, M. Goyal, Role of imaging in current acute ischemic stroke workflow for endovascular therapy. Stroke, 2015; 46:1453-61.

[21] S. Suzuki, J.L. Saver, P. Scott et al. Access to intra-arterial therapies for acute ischemic stroke: an analysis of the US population. AJNR Am J Neuroradiol, 2004; 25:1802-6.

[22] A.A. Tarnutzer, S.H. Lee, K.A. Robinson, Z. Wang, J.A. Edlow, D.E. Newman-Toker, ED misdiagnosis of cerebrovascular events in the era of modern neuroimaging: A meta-analysis. Neurology, 2017; 88:1468-77.

[23] Boehringer Ingelheim Limited. Actilyse. Summary of Product Characteristics, 2017. www.medicines.org.uk/emc/medicine/308. September 26, 2017.

[24] E.C. Jauch, J.L. Saver, H.P. Adams Jr et al; American Heart Association Stroke Council; Council on Cardiovascular Nursing; Council on Peripheral Vascular Disease; Council on Clinical Cardiology. Guidelines for the early management of patients with acute ischemic stroke: a guideline for healthcare professionals from the American Heart Association/American Stroke Association. Stroke, 2013; 44:870947.

[25] European Stroke Organisation (ESO) Executive Committee; ESO Writing Committee. Guidelines for management of ischaemic stroke and transient ischaemic attack 2008. Cerebrovasc Dis, 2008; 25:457507.

[26] S. Walter, P. Kostopoulos, A. Haass et al. Diagnosis and treatment of patients with stroke in a mobile stroke unit versus in hospital: a randomised controlled trial. Lancet Neurol, 2012; 11:397-404.

[27] A. Itrat, A. Taqui, R. Cerejo et al; Cleveland Pre-Hospital Acute Stroke Treatment Group. Telemedicine in prehospital stroke evaluation and thrombolysis: taking stroke treatment to the doorstep. JAMA Neurol, 2016; 73:162-8.

[28] M. Ebinger, B. Winter, M. Wendt et al; STEMO Consortium. Effect of the use of ambulance-based thrombolysis on time to thrombolysis in acute ischemic stroke: a randomized clinical trial. JAMA, 2014; 311:1622-31.

[29] R. Bowry, S. Parker, S.S. Rajan, et al. Benefits of stroke treatment using a Mobile Stroke Unit compared with standard management: the BEST-MSU Study Run-In Phase. Stroke, 2015; 46:3370-4.

[30] K. Fassbender, J.C. Grotta, S. Walter, I.Q. Grunwald, A. Ragoschke-Schumm, J.L. Saver, Mobile stroke units for prehospital thrombolysis, triage, and beyond: benefits and challenges. Lancet Neurol, 2017, $16: 227-37$. 